\title{
Review Article \\ Treatment and Prevention of Osteoarthritis through Exercise and Sports
}

\author{
Victor Valderrabano ${ }^{1,2}$ and Christina Steiger ${ }^{1,2}$ \\ ${ }^{1}$ Orthopaedic Department, University of Basel, Spitalstr. 21, 4031 Basel, Switzerland \\ ${ }^{2}$ Osteoarthritis Research Group, University of Basel, 4003 Basel, Switzerland \\ Correspondence should be addressed to Victor Valderrabano, vvalderrabano@uhbs.ch
}

Received 18 September 2010; Accepted 1 November 2010

Academic Editor: Iris Reuter

Copyright ( $) 2011$ V. Valderrabano and C. Steiger. This is an open access article distributed under the Creative Commons Attribution License, which permits unrestricted use, distribution, and reproduction in any medium, provided the original work is properly cited.

\begin{abstract}
Osteoarthritis $(\mathrm{OA})$ is a degenerative joint disease with a high prevalence among older people. To date, the pathogenesis of the disease and the link between muscle function and $\mathrm{OA}$ is not entirely understood. As there is no known cure for OA, current research focuses on prevention and symptomatic treatment of the disorder. Recent research has indicated that muscle weakness precedes the onset of OA symptoms. Furthermore, several studies show a beneficial effect of land-based aerobic and strengthening exercises on pain relief and joint function. Therefore, current research focuses on the possibility to employ exercise and sports in the prevention and treatment of OA.
\end{abstract}

\section{Introduction}

Osteoarthritis $(\mathrm{OA})$ is a chronic degenerative joint disorder having a significant economic impact on our health system world wide. Osteoarthritis is known to be most frequent in the lower extremity, especially the knee joint, where pathological joint impact and shear forces as well as posttraumatic risk factors cause early cartilage degeneration and "joint aging" [1]. The predominant symptoms are pain, a decreased joint range of motion (ROM) and stiffness, periarticular muscle weakness and atrophy, joint effusion and swelling, and physical disability. Despite the high prevalence of the disease, the pathogenesis and the reasons for progression are not entirely understood: primary OA versus secondary OA, as by, for example, inflammatory diseases and posttraumatic entities. Commonly, OA is characterised by structural changes of the entire joint. Partial to full thickness loss of articular cartilage, subchondral bone sclerosis, osteophyte formation, and thickening of the capsule are the typical clinical and radiological signs. Although radiological changes of OA constantly progress with age, clinical features do not necessarily correlate with radiological findings [2]. As there is no cure for OA, besides different symptoms-reducing drugs as well as joint preserving and replacement surgery, potentially amenable factors in the prevention and treatment of the disease such as muscle function are investigated. It is generally accepted that muscle weakness in OA is due to its atrophy, which is believed to be secondary to joint pain (arthrogenic muscle inhibition) $[3,4]$. Recent studies, however, suggested that age-related decrease of muscle volume is a risk factor for OA [5-11]. Thus, as muscle weakness could be targeted by a strengthening program, the question has been raised whether exercise and sports can be used to prevent and symptomatically treat OA.

\section{Muscular Dysbalance and Atrophy as a Cause of OA}

Muscles play an important role in joint biomechanics as they produce movement, absorb loading, and provide dynamic joint stability, and thus they are involved in the joint adaptation and degeneration process of OA $[6,7,12-16]$. Several studies have investigated the effect of muscular weakness and imbalance on the induction of OA [5-11, 17]. It is commonly accepted that, due to age or previous trauma, muscles are less intensively used. Such weak muscles do not only fatigue more rapidly, but they also display a slower 
voluntary and reflex motor control. Thus, once the protective muscular control is lost, excessive joint movement and instability with pathological shear and peak joint forces occur. As a consequence, stress-induced microtrauma to the articular cartilage leads to cartilage degeneration, pathological subchondral pressure increase, consequent subchondral bone sclerosis, and consequent joint collaps with axis maldeviation leading to the typical pathobiomechanical OA vicious circle. Herzog and Longino investigated the relationship of muscular dysfunction and the development of OA in a rabbit model [18]. In their study, knee extensor muscles of rabbits were injected with Botulinum type-A toxin causing a substantial reduction in muscle strength while maintaining functionality, thereby reproducing the clinical picture seen in patients with quadriceps weakness. Histological analyses of joint sections revealed signs of joint degeneration in two out of five animals sacrificed 4 weeks after injection with BTXA, indicating that even a short period of muscle weakness might be a risk factor for OA. In the development of knee joint OA, quadriceps weakness has been suggested to be a risk factor [5-7]. In this regard, a prospective study was carried out by Slemenda et al. investigating the occurrence of OA in women in a specified timeframe. His results showed that women developing OA had reduced knee extensor strength when compared to unaffected participants of the study [7]. A recent investigation by Valderrabano et al. reported similar results in patients with unilateral ankle OA. The authors were able to show that patients with ankle OA display a reduced calf circumference and had reduced mean electromyography frequencies of lower leg muscles on the affected side [4]. A recent followup study carried out in our institution included MRI analyses of lower leg muscle size. Here, it was determined that it were specific muscle groups which showed signs of OA muscle atrophy (unpublished data).

To determine the cellular abnormalities of muscle weakness and atrophy in OA, several histological and histochemical studies have been carried out $[4,19,20]$. Human muscle biopsy studies have shown that immobilisation in the healthy individual leads to atrophy of mainly type 1 fibres. In kneeOA patients, however, Nakamura and Suzuki found a type 2 fibre atrophy in the vastus lateralis [20]. In contrast to the slow-twitch type 1 fibres, these type 2 fibres are fast-twitch type fibres containing high levels of glycogen and enzymes and thus allow for more sustained tension. A recent study by Fink et al. also investigated the structural changes of the vastus medialis muscle in OA of the knee. Consistent with previous data by Nakamura, he found an atrophy of type 2 fibres in all specimens. Interestingly though, he also showed an additional type 1 fibre atrophy in $32 \%$ of patients [19]. As we all know, both type 2 and type 1 muscle fibres have been shown to increase in diameter upon muscle training [21-24]. Thus, the authors strongly believe that exercise programs and sports activity may be able to prevent or counteract muscle atrophy and thus prolong the onset of OA.

Lately, age-related muscle atrophy, namely sarcopenia, has been frequently discussed in literature. This multifactorial disease has been recognised as an important geriatric syndrome [25]. It is characterised by a generalised loss of skeletal muscle mass leading to a significant decrease in strength and can be distinguished on a cellular level from disuse muscle atrophy [26-28]. Various mechanisms such as endocrine dysfunctions, neurodegenerative disease and inadequate nutrition but also muscle disuse and immobility are thought to contribute to its onset and progression. Although sarcopenia mainly affects older people, it has also been found in younger adults. Current treatment concepts include exercise programs to recover some muscle mass and increase strength [25-28]. As patients with sarcopenia display reduced muscle mass and strength and thus are less able to control joint motion, it is likely they have a high risk for developing OA.

To date, many questions regarding the pathogenesis of OA remain open. However, muscular weakness and in some cases even muscular atrophy, either preceding or accompanying the OA disease, is frequently observed. As muscle increases in size upon exercise, atrophy might not only be a result of joint stiffness and pain but also by age-related sarcopenia and physical immobilisation and reduction of sports activity. Since no cure is available for OA, current research should focus on the prevention of this age-related joint disorder. Muscle weakness and atrophy contribute to the OA process, and thus the question has been raised whether exercise and sports could be employed to prolong time to pain onset or relieve pain and improve quality of life in symptomatic patients.

In summary, a strong correlation between muscle atrophy and osteoarthritis has been found on many occasions. It is currently believed that muscle atrophy regardless of causative pathomechanism is a major contributing factor for the development of OA. As exercise increases muscle mass and improves muscle function, it might play a crucial role in the prevention and treatment of OA.

\section{Exercise and Sports in the Treatment of OA}

As there is no cure for OA, treatments currently focus on management of symptoms. Pain relief, improved joint function, and joint stability are the main goals of therapy. Studies conducted within recent years provided data that supports the assumption that muscle weakness and muscle atrophy contribute to the disease process [5-11]. Thus, rehabilitation and physiotherapy were often prescribed with the intention to alleviate pain and increase mobility. However, as exercise has to be performed on a regular basis in order to counteract muscle atrophy, continuous exercise programs are recommended in people with degenerative joint disease. Therapeutic exercise regimes either focus on muscle strengthening and stretching exercises or on aerobic activity which can be land or water based. To verify the effectiveness of such physical activity, several studies [29-35] as well as a few meta-analyses [36-42] have been carried out. No major difference in effectiveness was reported between land-based aerobic and strengthening exercise [40-42]. Concerning the beneficial effects of aquatic-based exercise, controversial data exists. In one study, aquatic exercise has been shown to be less efficient in reducing pain and improving muscle function than land-based exercise [32]. A review by 
Bartels analysed data from four different studies and could show short-term improvement of OA symptoms in knee OA patients supposedly due to an increase of strength of the muscles around the knee. However, long-term effects could not be verified [36]. A recent study by Cadmus reported beneficial effects of aquatic-based exercise only for obese patients but not for nonobese [29]. Due to the buoyancy of water, it is possible to exercise without experiencing full body weight allowing for relatively pain-free motion. Thus, aquatic-based exercise might be a good way to introduce disabled and obese patients to sports. A frequently raised question concerns the intensity of exercise. After reviewing several aerobic exercise studies, Brosseau concluded that both low- and high-intensity aerobic exercise was beneficial in patients with OA [37]. Jan et al. investigated the effects of high and low resistance training and the effects of weightbearing versus nonweight-bearing exercise in patients with $\mathrm{OA}$ of the knee. He determined that both low- and highresistance type training led to reduced pain and improved function. A recorded increased walking speed on uneven terrains led the authors to speculate that both types of resistance training improved balance and proprioception. Albeit not statistically significant, high resistance training also demonstrated consistently higher functional performance and greater reduction of pain [31]. Furthermore, when comparing the effects of weight-bearing and nonweight-bearing exercises, an improved position sense was found in addition to decreased pain scores and increased muscular strength [30]. A recent study investigated the effects of Tai Chi in treating OA of the knee. This traditional Chinese mind-body exercise combines strength, balance, and flexibility training. When compared to a wellness education and stretching program, the overall outcome showed a greater improvement of pain and physical function in patients attending the Tai Chi training [35].

In conclusion, literature shows clear improvement of OA symptoms in patients undertaking exercise programs. Decreased pain and increased muscle function have been reported for both strengthening and aerobic exercises. However, these effects do not persist if exercise programs are discontinued. Thus, the motivation of the patient to start and continuously practice exercise is of crucial importance. Many patients suffering from OA refuse to start exercising due to joint pain. In such cases, the use of painkillers during the first weeks of an exercise program might not only facilitate joint movement but can also drastically improve patient compliance. Furthermore, in case of severe joint pain or in obese patients, the authors believe that an initial period of water-based exercise is helpful. As swimming or aqua jogging provides a muscle workout without joint loading, further pain and weight-related joint destruction is avoided. Although many studies investigated the effect of different exercise types, hardly any study can be found investigating the effect of different kinds of sports on OA. Current knowledge supports practicing sports which avoid sudden peak stresses but improve muscle function and contribute to the stabilisation of the affected joints. Sports that fulfil these criteria and are regularly recommended to people suffering from OA are low-impact sports, as cycling, nordic walking, and aquajogging. However, data on the short- and longterm effect of theses types of sport in OA are scarce, and therefore further studies are urgently needed. When it comes to exercise programs, both low- and high-resistance training with or without weight bearing has been shown to have beneficial effects. Patients unable to participate in exercise or sport programs should be encouraged to continue carrying out activities of daily life such as walking the dog, gardening, or biking to work. However, it is the authors' opinion that exercise and sports can and should be practiced at any stage of $\mathrm{OA}$ although the intensity has to be adjusted to the individual capability.

\section{Exercise and Sports in the Prevention of OA}

Exercise has been shown to improve pain and function in OA [38] and is recommended by the Osteoarthritis Research Society International (OARSI) for the management of hip and knee OA [43]. However, to date, very little research has been conducted to investigate whether exercise and sports can also be used to prevent the onset of OA. It is widely recognised that physical activity is beneficial to cardiovascular health and is considered an integral component of a healthy lifestyle [44]. Furthermore, regular exercise facilitates weight loss and prevents weight gain. As obesity is a recognised risk factor for knee OA $[45,46]$, exercise is thought to have a beneficial effect on the OA disease progression. However, OA is not only found in obese patients and not all joints are affected equally by weight increase [47]. Thus, other diseaserelated factors have been investigated. Muscle weakness and muscle atrophy have been reported to occur even before the onset of symptomatic OA [6]. As muscle weakness usually results from disuse of muscles, the question has been raised whether exercise and sports could be employed to prevent or counteract muscle weakness and therefore prevent or delay the onset of OA. Recently, two studies have been published on the protective effect of exercise and of quadriceps strength in the prevention of cartilage loss at the tibiofemoral joint $[48,49]$. Amin et al. [48] showed that there was no association between a decrease in quadriceps strength and cartilage loss in a 30 months trial. However, he also determined that participants of the study with greatest quadriceps strength displayed the least cartilage loss at the lateral compartment of the patellofemoral joint indicating that a strong quadriceps muscle had protective cartilage effects. In the study by Otterness et al. [49], the effects of daily exercise on cartilage degeneration in hamsters were investigated. Hamsters were undergoing either 3 months of daily exercise or 3 months of sedentary living. Histologic analyses of the femoral articular cartilage of the exercising hamsters showed a smooth surface similar to that of young control animals. In contrast, signs of cartilage degeneration were seen in sedentary hamsters. Furthermore, cartilage composition in these animals showed a reduced proteoglycan content and synovial fluid volume. This data indicates that at least in hamsters early cartilage degeneration is not a result of exercise but rather of inactivity and decreased muscle function. Thus, it can be argued that inactivity not only 
promotes muscle weakness and thereby joint instability but also makes cartilage more prone to damage by altering its structure. As the data by Otterness was derived in a hamster model, it was questionable whether the same cartilage change would occur in humans. A literature search revealed a study in human in which the effects of moderate exercise on cartilage were analysed in knees prone to develop OA [50]. The study participants had all undergone partial medial meniscectomy 3 to 5 years previously and were either asked to complete a 4-month exercise program or remain at their current physical exercise level. At the end of the study, $68 \%$ of the exercise group reported an increase in activity level. Furthermore, glycosaminoglycan levels which were measured by $\mathrm{T} 1$ relaxation time on MRI scans were clearly increased, indicating that exercise also has a beneficial direct effect on cartilage structure in human. The exact mechanism that causes chondroprotection upon exercise however is not entirely understood. A recent study discovered an increase in IL10 levels of synovial fluid after acute resistance exercise in patients with OA of the knee [51]. IL10 is an antiinflammatory cytokine with chondroprotective properties. It has been shown to suppress the release of inflammatory cytokines by macrophages and activates chondrocytes and synoviocytes and, thereby, is interfering with important steps in the pathogenesis of OA $[52,53]$. One could speculate that during regular exercise, IL10 levels would be constantly elevated, thus reducing inflammation in the joint and hence reducing pain. As a result, physical activity can be undertaken more frequently, thus increasing muscle mass and thereby providing more joint stability. However, to confirm this hypothesis a lot more research is needed.

In summary, investigations on the effectiveness of exercise in the prevention of OA have revealed some interesting facts. Animal studies and clinical trials clearly showed a protective effect of exercise on joint cartilage, thus perhaps reducing the likelihood for developing OA. Furthermore, an anti-inflammatory environment was found in OA knee joints upon exercise. Assuming that the same environment exists in a healthy joint under the same conditions exercise might be very effective in preventing the onset of disease. However, to fully understand the protective effects of exercise on the development of OA, further clinical and molecular studies are needed.

\section{Conclusions}

As overall conclusion, $\mathrm{OA}$ is a multifactorial disease with a pathogenic link to muscle function and volume that is not entirely understood. Over the last decade, research has provided data supporting the hypothesis that exercise is not the reason for OA but rather delays onset and alleviates symptoms of the disorder. Furthermore, several studies have shown that muscle weakness is a predisposing factor for $\mathrm{OA}$ and that muscle atrophy is a common finding in OA. Changes in cartilage structure have also been investigated, and it was determined that in order to remain firm a certain amount of muscle exercise was needed. Taken into account all the above mentioned data, this would argue that exercise and sports should be used in the prevention and also in the treatment of OA and aging people. To determine which type of exercise would be most appropriate, various studies were carried out. It was shown that aquatic exercise was beneficial only in the beginning and in obese people whereas landbased aerobic and strengthening exercises had a continuous effect for as long as they were carried out. Once exercise is discontinued, all the beneficial effects that come with it vanish as well. Thus, the authors strongly believe that any additional measures that help to motivate patients to continue sports, such as training schedules, group exercises, or instructional sessions with physiotherapists are of crucial importance. To date, many studies have been carried out investigating the effect of exercise on the progression of OA. Although alleviation of pain has been shown to occur upon regular exercise, the minimal intensity of training necessary for such a positive effect still needs to be determined. Therefore, one of the biggest challenges still remains to understand the pathogenesis of OA in order to prevent the onset or find further treatments for this debilitating disorder.

\section{References}

[1] D. T. Felson, A. Naimark, J. Anderson, L. Kazis, W. Castelli, and R. F. Meenan, "The prevalence of knee osteoarthritis in the elderly. The Framingham Osteoarthritis Study," Arthritis and Rheumatism, vol. 30, no. 8, pp. 914-918, 1987.

[2] A. A. M. C. Claessens, J. S. A. G. Schouten, F. A. van den Ouweland, and H. A. Valkenburg, "Do clinical findings associate with radiographic osteoarthritis of the knee?" Annals of the Rheumatic Diseases, vol. 49, no. 10, pp. 771-774, 1990.

[3] V. Valderrabano, B. M. Nigg, V. von Tscharner, D. J. Stefanyshyn, B. Goepfert, and B. Hintermann, "Gait analysis in ankle osteoarthritis and total ankle replacement," Clinical Biomechanics, vol. 22, no. 8, pp. 894-904, 2007.

[4] V. Valderrabano, V. von Tscharner, B. M. Nigg et al., "Lower leg muscle atrophy in ankle osteoarthritis," Journal of Orthopaedic Research, vol. 24, no. 12, pp. 2159-2169, 2006.

[5] S. Ikeda, H. Tsumura, and T. Torisu, "Age-related quadricepsdominant muscle atrophy and incident radiographic knee osteoarthritis," Journal of Orthopaedic Science, vol. 10, no. 2, pp. 121-126, 2005.

[6] C. Slemenda, K. D. Brandt, D. K. Heilman et al., "Quadriceps weakness and osteoarthritis of the knee," Annals of Internal Medicine, vol. 127, no. 2, pp. 97-104, 1997.

[7] C. Slemenda, D. K. Heilman, K. D. Brandt et al., "Reduced quadriceps strength relative to body weight: a risk factor for knee osteoarthritis in women?" Arthritis and Rheumatism, vol. 41, no. 11, pp. 1951-1959, 1998.

[8] A. Amaro, F. Amado, J. A. Duarte, and H. J. Appell, "Gluteus medius muscle atrophy is related to contralateral and ipsilateral hip joint osteoarthritis," International Journal of Sports Medicine, vol. 28, no. 12, pp. 1035-1039, 2007.

[9] A. Grimaldi, C. Richardson, G. Durbridge, W. Donnelly, R. Darnell, and J. Hides, "The association between degenerative hip joint pathology and size of the gluteus maximus and tensor fascia lata muscles," Manual Therapy, vol. 14, no. 6, pp. 611617, 2009.

[10] A. Grimaldi, C. Richardson, W. Stanton, G. Durbridge, W. Donnelly, and J. Hides, "The association between degenerative hip joint pathology and size of the gluteus medius, gluteus 
minimus and piriformis muscles," Manual Therapy, vol. 14, no. 6, pp. 605-610, 2009.

[11] O. D. Schipplein and T. P. Andriacchi, "Interaction between active and passive knee stabilizers during level walking," Journal of Orthopaedic Research, vol. 9, no. 1, pp. 113-119, 1991.

[12] K. D. Brandt, "Putting some muscle into osteoarthritis," Annals of Internal Medicine, vol. 127, no. 2, pp. 154-156, 1997.

[13] T. Hortobágyi, J. Garry, D. Holbert, and P. Devita, "Aberrations in the control of quadriceps muscle force in patients with knee osteoarthritis," Arthritis Care and Research, vol. 51, no. 4, pp. 562-569, 2004.

[14] T. Hortobágyi, L. Westerkamp, S. Beam et al., "Altered hamstring-quadriceps muscle balance in patients with knee osteoarthritis," Clinical Biomechanics, vol. 20, no. 1, pp. 97104, 2005.

[15] M. D. Lewek, K. S. Rudolph, and L. Snyder-Mackler, "Quadriceps femoris muscle weakness and activation failure in patients with symptomatic knee osteoarthritis," Journal of Orthopaedic Research, vol. 22, no. 1, pp. 110-115, 2004.

[16] A. E. Mikesky, A. Meyer, and K. L. Thompson, "Relationship between quadriceps strength and rate of loading during gait in women," Journal of Orthopaedic Research, vol. 18, no. 2, pp. $171-175,2000$.

[17] S. C. O’Reilly, A. Jones, K. R. Muir, and M. Doherty, "Quadriceps weakness in knee osteoarthritis: the effect on pain and disability," Annals of the Rheumatic Diseases, vol. 57, no. 10, pp. 588-594, 1998.

[18] W. Herzog and D. Longino, "The role of muscles in joint degeneration and osteoarthritis," Journal of Biomechanics, vol. 40, supplement 1, pp. S54-S63, 2007.

[19] B. Fink, M. Egl, J. Singer, M. Fuerst, M. Bubenheim, and E. Neuen-Jacob, "Morphologic changes in the vastus medialis muscle in patients with osteoarthritis of the knee," Arthritis and Rheumatism, vol. 56, no. 11, pp. 3626-3633, 2007.

[20] T. Nakamura and K. Suzuki, "Muscular changes in osteoarthritis of the hip and knee," Journal of the Japanese Orthopaedic Association, vol. 66, no. 5, pp. 467-475, 1992.

[21] D. L. Costill, W. J. Fink, and M. L. Pollock, "Muscle fiber composition and enzyme activities of elite distance runners," Medicine and Science in Sports and Exercise, vol. 8, no. 2, pp. 96-100, 1976.

[22] W. J. Fink, D. L. Costill, and M. L. Pollock, "Submaximal and maximal working capacity of elite distance runners. II. Muscle fiber composition and enzyme activities," Annals of the New York Academy of Sciences, vol. 301, pp. 323-327, 1977.

[23] J. M. Lüthi, C. Gerber, H. Claassen, and H. Hoppeler, "The injured and the immobilized muscle cell: ultrastructural observations," Sportverletzung Sportschaden, vol. 3, no. 2, pp. 58-61, 1989.

[24] B. Saltin, J. Henriksson, E. Nygaard, P. Andersen, and E. Jansson, "Fiber types and metabolic potentials of skeletal muscles in sedentary man and endurance runners," Annals of the New York Academy of Sciences, vol. 301, pp. 3-29, 1977.

[25] A. J. Cruz-Jentoft, J. P. Baeyens, J. M. Bauer et al., "Sarcopenia: European consensus on definition and diagnosis: report of the European Working Group on Sarcopenia in Older People," Age and Ageing, vol. 39, no. 4, pp. 412-423, 2010.

[26] B. H. Goodpaster, S. W. Park, T. B. Harris et al., "The loss of skeletal muscle strength, mass, and quality in older adults: the health, aging and body composition study," Journals of Gerontology A, vol. 61, no. 10, pp. 1059-1064, 2006.
[27] I. Janssen, R. N. Baumgartner, R. Ross, I. H. Rosenberg, and R. Roubenoff, "Skeletal muscle cutpoints associated with elevated physical disability risk in older men and women," American Journal of Epidemiology, vol. 159, no. 4, pp. 413-421, 2004.

[28] T. E. Jones, K. W. Stephenson, J. G. King, K. R. Knight, T. L. Marshall, and W. B. Scott, "Sarcopenia-mechanisms and treatments," Journal of Geriatric Physical Therapy, vol. 32, no. 2, pp. 39-45, 2009.

[29] L. Cadmus, M. B. Patrick, M. L. Maclejewski, T. Topolski, B. Belza, and D. L. Patrick, "Community-based aquatic exercise and quality of life in persons with osteoarthritis," Medicine and Science in Sports and Exercise, vol. 42, no. 1, pp. 8-15, 2010.

[30] M. H. Jan, C. H. Lin, Y. F. Lin, J. J. Lin, and D. H. Lin, "Effects of weight-bearing versus nonweight-bearing exercise on function, walking speed, and position sense in participants with knee osteoarthritis: a randomized controlled trial," Archives of Physical Medicine and Rehabilitation, vol. 90, no. 6, pp. 897-904, 2009.

[31] M. H. Jan, J. J. Lin, J. J. Liau, Y. F. Lin, and D. H. Lin, "Investigation of clinical effects of high- and low-resistance training for patients with knee osteoarthritis: a randomized controlled trial," Physical Therapy, vol. 88, no. 4, pp. 427-436, 2008.

[32] H. Lund, U. Weile, R. Christensen et al., "A randomized controlled trial of aquatic and land-based exercise in patients with knee osteoarthritis," Journal of Rehabilitation Medicine, vol. 40, no. 2, pp. 137-144, 2008.

[33] A. E. Mikesky, S. A. Mazzuca, K. D. Brandt, S. M. Perkins, T. Damush, and K. A. Lane, "Effects of strength training on the incidence and progression of knee osteoarthritis," Arthritis Care and Research, vol. 55, no. 5, pp. 690-699, 2006.

[34] B. W. J. H. Penninx, S. P. Messier, W. J. Rejeski et al., "Physical exercise and the prevention of disability in activities of daily living in older persons with osteoarthritis," Archives of Internal Medicine, vol. 161, no. 19, pp. 2309-2316, 2001.

[35] C. Wang, C. H. Schmid, P. L. Hibberd et al., "Tai Chi is effective in treating knee osteoarthritis: a randomized controlled trial," Arthritis Care and Research, vol. 61, no. 11, pp. 1545-1553, 2009.

[36] E. M. Bartels, H. Lund, K. B. Hagen, H. Dagfinrud, R. Christensen, and B. Danneskiold-Samsøe, "Aquatic exercise for the treatment of knee and hip osteoarthritis," Cochrane Database of Systematic Reviews, no. 4, Article ID CD005523, 2007.

[37] L. Brosseau, L. MacLeay, V. Robinson, G. Wells, and P. Tugwell, "Intensity of exercise for the treatment of osteoarthritis," Cochrane Database of Systematic Reviews, no. 2, Article ID CD004259, 2003.

[38] M. Fransen and S. McConnell, "Exercise for osteoarthritis of the knee," Cochrane Database of Systematic Reviews, no. 4, Article ID CD004376, 2008.

[39] M. Fransen, S. McConnell, and M. Bell, "Exercise for osteoarthritis of the hip or knee," Cochrane Database of Systematic Reviews, no. 3, Article ID CD004286, 2003.

[40] M. Fransen, S. McConnell, and M. Bell, “Therapeutic exercise for people with osteoarthritis of the hip or knee. A systematic review," Journal of Rheumatology, vol. 29, no. 8, pp. 1737-1745, 2002.

[41] L. E. Hart, D. A. Haaland, D. A. Baribeau, I. M. Mukovozov, and T. F. Sabljic, "The relationship between exercise and osteoarthritis in the elderly," Clinical Journal of Sport Medicine, vol. 18 , no. 6, pp. 508-521, 2008. 
[42] E. Roddy, W. Zhang, and M. Doherty, "Aerobic walking or strengthening exercise for osteoarthritis of the knee? A systematic review," Annals of the Rheumatic Diseases, vol. 64, no. 4, pp. 544-548, 2005.

[43] W. Zhang, R. W. Moskowitz, G. Nuki et al., "OARSI recommendations for the management of hip and knee osteoarthritis, part II: OARSI evidence-based, expert consensus guidelines," Osteoarthritis and Cartilage, vol. 16, no. 2, pp. 137-162, 2008.

[44] R. R. Pate, M. Pratt, S. N. Blair et al., "Physical activity and public health: a recommendation from the Centers for Disease Control and Prevention and the American College of Sports Medicine," Journal of the American Medical Association, vol. 273, no. 5, pp. 402-407, 1995.

[45] D. J. Hart, D. V. Doyle, and T. D. Spector, "Incidence and risk factors for radiographic knee osteoarthritis in middle-aged women: the Chingford study," Arthritis and Rheumatism, vol. 42, no. 1, pp. 17-24, 1999.

[46] J. H. Kellgren, J. S. Lawrence, and F. Bier, "Genetic factors in generalized osteo-arthrosis," Annals of the Rheumatic Diseases, vol. 22, pp. 237-255, 1963.

[47] P. D. Saville and J. Dickson, "Age and weight in osteoarthritis of the hip," Arthritis and Rheumatism, vol. 11, no. 5, pp. 635644, 1968.

[48] S. Amin, K. Baker, J. Niu et al., "Quadriceps strength and the risk of cartilage loss and symptom progression in knee osteoarthritis," Arthritis and Rheumatism, vol. 60, no. 1, pp. 189-198, 2009.

[49] I. G. Otterness, J. D. Eskra, M. L. Bliven, A. K. Shay, J. P. Pelletier, and A. J. Milici, "Exercise protects against articular cartilage degeneration in the hamster," Arthritis and Rheumatism, vol. 41, no. 11, pp. 2068-2076, 1998.

[50] E. M. Roos and L. Dahlberg, "Positive effects of moderate exercise on glycosaminoglycan content in knee cartilage: a four-month, randomized, controlled trial in patients at risk of osteoarthritis," Arthritis and Rheumatism, vol. 52, no. 11, pp. 3507-3514, 2005.

[51] I. C. Helmark, U. R. Mikkelsen, J. Borglum et al., "Exercise increases interleukin-10 levels both intraarticularly and perisynovially in patients with knee osteoarthritis: a randomized controlled trial," Arthritis Research \& Therapy, vol. 12, article R126, 2010.

[52] P. H. Hart, M. J. Ahern, M. D. Smith, and J. J. FinlayJones, "Comparison of the suppressive effects of interleukin10 and interleukin-4 on synovial fluid macrophages and blood monocytes from patients with inflammatory arthritis," Immunology, vol. 84, no. 4, pp. 536-542, 1995.

[53] G. Schulze-Tanzil, H. Zreiqat, R. Sabat et al., "Interleukin-10 and articular cartilage: experimental therapeutical approaches in cartilage disorders," Current Gene Therapy, vol. 9, no. 4, pp. 306-315, 2009. 


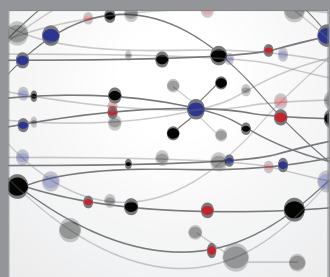

The Scientific World Journal
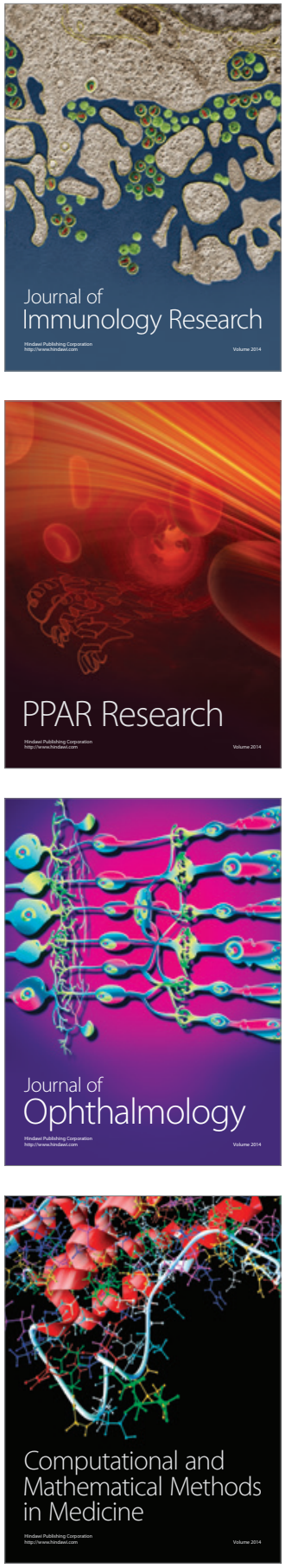

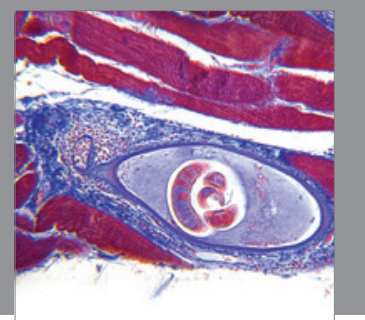

Gastroenterology

Research and Practice
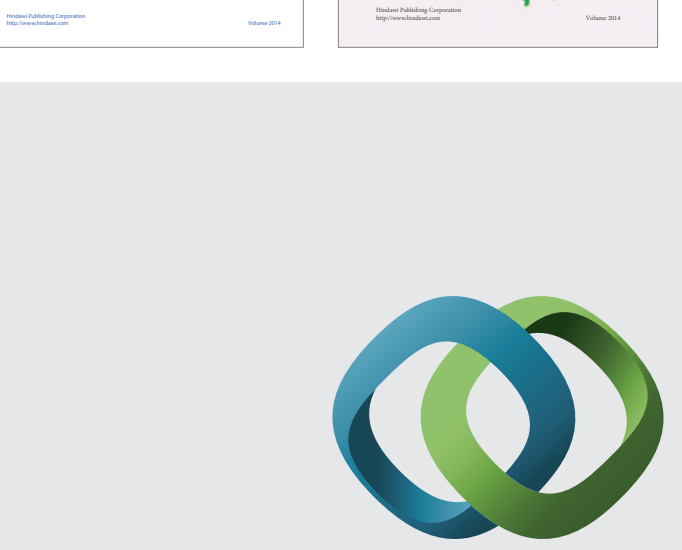

\section{Hindawi}

Submit your manuscripts at

http://www.hindawi.com
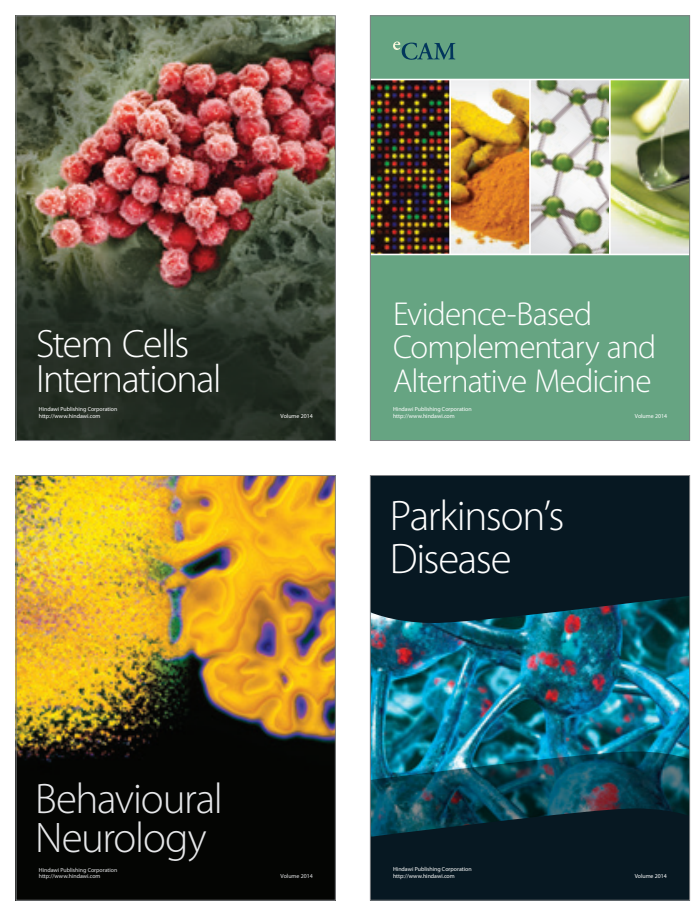

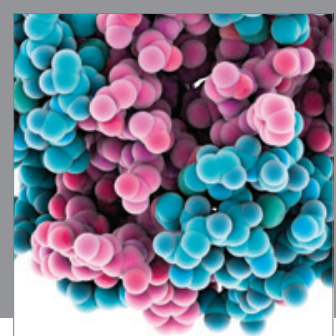

Journal of
Diabetes Research

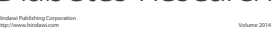

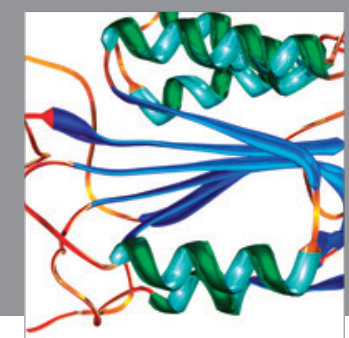

Disease Markers
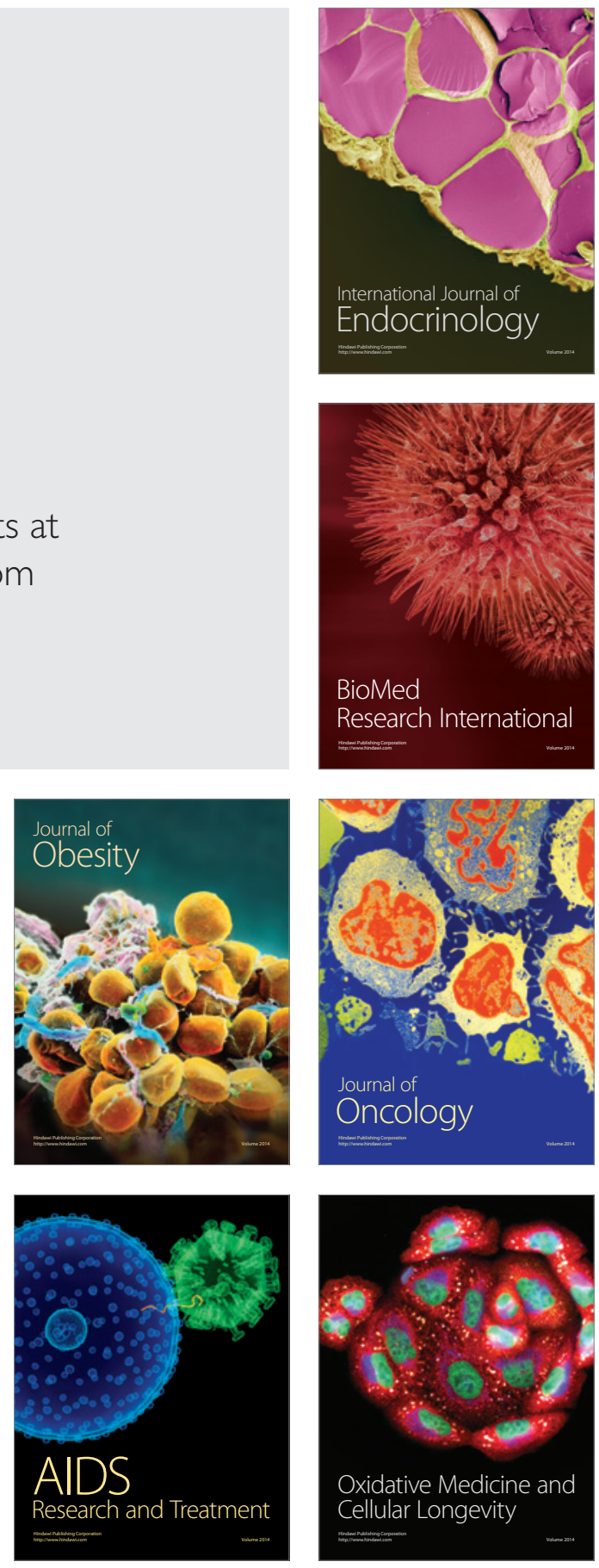